\title{
Evolution of the Warm-Core Structure during the Eyewall Replacement Cycle in a Numerically Simulated Tropical Cyclone
}

\author{
HUI WANG \\ State Key Laboratory of Severe Weather, Chinese Academy of Meteorological Sciences, Beijing, China \\ YUQING WANG \\ State Key Laboratory of Severe Weather, Chinese Academy of Meteorological Sciences, Beijing, China, \\ and International Pacific Research Center, and Department of Atmospheric Sciences, \\ University of Hawai' $i$ at Mānoa, Honolulu, Hawaii \\ JING XU AND YIHONG DUAN \\ State Key Laboratory of Severe Weather, Chinese Academy of Meteorological Sciences, Beijing, China
}

(Manuscript received 26 January 2019, in final form 4 June 2019)

\begin{abstract}
This study examines the evolution of the warm-core structure during the secondary eyewall formation (SEF) and the subsequent eyewall replacement cycle (ERC) in a numerically simulated tropical cyclone (TC) under idealized conditions. Results show that prior to the SEF, the TC exhibited a double warm-core structure centered in the middle and upper troposphere in the eye region, and as the storm intensified with a rapid outward expansion of tangential winds, the warm core strengthened and a secondary off-center warm ring developed between 8- and 16-km heights near the outer edge of the eye. During the SEF, both the upper-level warm core and the secondary off-center warm ring rapidly strengthened. As the secondary eyewall intensified and contracted and the primary eyewall weakened and dissipated, the off-center warm ring extended inward and merged with the inner warm core to form a warm core typical of a single-eyewall TC. Results from the azimuthal-mean potential temperature budget indicate that the warming in the eye is due to subsidence and the warming above 14-km height outside the eye is largely contributed by radial warm advection in the outflow. The development of the off-center warm ring is largely due to the subsidence warming near the inner edge of the primary eyewall and in the moat area and the warming by diabatic heating in the upper part of the inner eyewall below 14-km height. Further analysis indicates that the eddy advection also played some role in the warming above 12-km height in the upper troposphere.
\end{abstract}

\section{Introduction}

The secondary eyewall formation (SEF) and the following eyewall replacement cycle (ERC) in a tropical cyclone (TC) have been investigated extensively in the literature because of their significant impacts on TC structure, size, and intensity changes. Most previous studies have focused on the formation mechanisms of the secondary convective ring structure and the establishment of the secondary tangential wind maximum based on observations and high-resolution numerical simulations (Willoughby et al. 1982; Terwey and Montgomery 2008; Wang 2009; Judt and Chen 2010; Qiu

Corresponding author: Prof. Yuqing Wang, yuqing@hawaii.edu et al. 2010; Qiu and Tan 2013; Abarca and Corbosiero 2011; Huang et al. 2012; Bell et al. 2012; Rozoff et al. 2012; Sun et al. 2013; Abarca and Montgomery 2013, 2014; Kepert 2013; Zhu and Zhu 2014; Wang et al. 2016; Zhang et al. 2017; Dai et al. 2017; Tyner et al. 2018; Didlake et al. 2018; Wang et al. 2019). Only a few studies have given attention to the thermodynamic conditions within the eye and the moat regions in TCs with concentric-eyewall structures, and the evolution of the warm-core structure during the ERC.

Houze et al. (2007) examined the ERC and the associated intensity change in Hurricane Rita (2005) based on aircraft data. They found that the temperature profile of the eye below 3-km height had little difference from that of the moat at the time during the SEF and ERC as 
the inner eyewall weakened and was gradually replaced by the outer eyewall. Sitkowski et al. (2011) studied the inner-core thermodynamic changes during the ERC in hurricanes using flight-level data. They found that both the inner-core and outer-core regions showed a warming trend during the ERC with the largest temperature increase observed in the moat region. Since the flight level was around $700 \mathrm{hPa}$, the collected flight-level data mainly reflected the mid- to lower-tropospheric thermodynamic evolution. However, the maximum warm core in strong TCs is most frequently located in the upper troposphere (Willoughby 1998; Wang and Jiang 2019) where most research aircraft could not reach. Similarly, the temperature data measured by dropsondes from aircraft in Hurricane Rita (2005) studied by Houze et al. (2007) mainly reflected the low-level thermodynamic features of the eye and moat regions as well. Nevertheless, these studies attributed the warming in the eye and the moat regions to the enhanced subsidence forced by convective heating in the strengthening outer eyewall.

Rozoff et al. (2008) studied the dynamical aspects of the ERC with the focus on the warm-core evolution based on the balanced vortex model. To allow for analytical solutions, they subdivided five radial regions with their individual inertial stability and parameterized diabatic heating rate, representing the eye, inner eyewall, moat, outer eyewall, and far field. Their analytical solution of the transverse circulation equation showed that in the mature concentric-eyewall configuration, subsidence and warming in the moat area were governed mainly by enhanced inertial stability and the subsidence associated with a strengthening outer eyewall. Their solution also demonstrated that diabatic heating in the inner eyewall and imbedded in a region of high inertial stability could induce larger warming than diabatic heating in the outer eyewall surrounded by the far-field region of low inertial stability. As a result, the increasing warming of the moat region during the ERC can be considered as a response to the enhancing inertial stability of the moat region as the outer eyewall contracts and intensifies. Results from Rozoff et al. (2008) thus strongly suggest that subsidence warming in the moat region contains comparable contributions from both inner and outer eyewalls but most of this subsidence results from enhanced inertial stability associated with a contracting and strengthening outer eyewall.

The warm-core structure of a single-eyewall TC has been comprehensively investigated through observational, theoretical, and modeling studies (e.g., Kurihara 1975; Smith 1980; Kossin and Eastin 2001; Vigh and Schubert 2009; Stern and Nolan 2012; Stern and Zhang 2013; Ohno and Satoh 2015). It is generally believed that the height and strength of the warm core are related to the intensity as well as the size and slope of the eyewall of the storm and controlled primarily by adiabatic subsidence warming in the eye region forced by eyewall convection (Eliassen 1951; Smith 1980; Schubert and Hack 1982; Hack and Schubert 1986; Nolan et al. 2007; Schubert et al. 2007; Wang 2012; Durden 2013). In addition, part of the warming may be contributed by the warm air from the lower stratosphere advected toward the eye region by radial inflow near the tropopause (Halverson et al. 2006; Zhang and Chen 2012; Chen and Zhang 2013; Wang and Wang 2014; Kieu et al. 2016; Komaromi and Doyle 2017). Stern and Zhang (2013) performed a potential temperature budget analysis of an idealized simulation and found that in addition to the adiabatic subsidence warming by the mean vertical advection, the eddy radial advection of potential temperature also played an important role in the early formation stage of the warm-core structure of their simulated TC. Ohno and Satoh (2015) performed a similar idealized simulation and potential temperature budget analysis and found that the largest positive contribution to the total warming is by the azimuthal-mean rather than the eddy component, in particular during the stage when the simulated TC was close to its peak intensity. The analyses of forced secondary circulation using the Sawyer-Eliassen equation indicated that the upperlevel subsidence in the eye was substantially enhanced when the TC circulation extended high enough to penetrate into the lower stratosphere.

Although the formation and maintenance of the warm core in a double-eyewall TC may share some common physical processes with those of a single-eyewall TC, some aspects could be different between the two distinct TCs as revealed in limited previous studies. In particular, the double-eyewall structure often forms after the TC reaches a relatively high intensity (e.g., Willoughby et al. 1982; Kossin and Eastin 2001), and thus a relatively stronger warm-core structure already existed before the formation of the secondary eyewall. Although some studies have examined the evolution of the warm-core structure in the double-eyewall TCs based on observations (Houze et al. 2007; Sitkowski et al. 2012) and theoretical framework (Rozoff et al. 2008), and many studies have investigated the SEF mechanisms through idealized and real-case simulations [see a review by Wang (2012)], few studies have examined the evolution of the warm-core structure during the SEF and ERC in any numerically simulated double-eyewall TCs.

This study provides an initial analysis on the evolution of the warm-core structure during the SEF and ERC in a simulated TC under idealized conditions recently studied by Wang et al. (2019). In Wang et al. (2019), the 
SEF mechanism of the simulated TC has been analyzed (see section 2 for an overview). They showed that the continuous downward and inward propagation and the subsequent axisymmetrization of asymmetric tangential winds in an outer rainband led to the spinup of the secondary tangential wind maximum and the SEF. As an extension of the study of Wang et al. (2019), this study will analyze the evolution of the warm-core structure prior to and during the SEF, and also analyze the ERC of the simulated TC and examine the controlling factors that affect the warm-core evolution by conducting a potential temperature budget analysis. Section 2 provides an overview of the simulated SEF and the subsequent ERC. Section 3 describes the evolution of the warm-core structure in the simulated TC. A potential temperature budget analysis is performed to highlight processes that control the evolution of the warm-core structure during the SEF and the ERC in the simulated $\mathrm{TC}$ in section 4. Main results are summarized in section 5 .

\section{An overview of the simulation, the SEF, and the subsequent ERC}

The simulation results discussed in this study were the same as those analyzed to understand the SEF processes in a simulated TC under idealized conditions in a quiescent environment on an $f$ plane of $20^{\circ} \mathrm{N}$ in Wang et al. (2019). The WRF Model (version 3.5.1) was used and configured with quadruply nested domains with their respective horizontal grid spacings of $45,15,5$, and $1.67 \mathrm{~km}$ and domain sizes of $200 \times 200,250 \times 250,268 \times 268$, and $388 \times 388$. The model had 50 vertical levels from the surface up to $50 \mathrm{hPa}$. The three inner nested meshes were initially located near the center of their parent domains and moved automatically following the model TC during the model integration. The model physics parameterizations included the WRF single-moment 6-class microphysics scheme (WSM6; Hong and Lim 2006), the Yonsei University (YSU) planetary boundary layer (PBL) scheme (Hong et al. 2006), the shortwave radiation scheme of Dudhia (Dudhia 1989), and the longwave radiation scheme of the Rapid Radiative Transfer Model (RRTM; Mlawer et al. 1997). The KainFritch cumulus parameterization scheme (Kain and Fritsch 1993) was applied only in the two outer meshes.

The model was initialized with an axisymmetric cyclonic vortex, which had the maximum tangential wind of $25 \mathrm{~m} \mathrm{~s}^{-1}$ at the radius of $80 \mathrm{~km}$. The initial tangential wind of the vortex decreased radially to zero at a radius of $1000 \mathrm{~km}$ and vertically to zero at $100 \mathrm{hPa}$ (Wang 2007). The unperturbed environment sounding was based on the western Pacific clear-sky environment of
Gray et al. (1975). The sea surface temperature (SST) was fixed at $29^{\circ} \mathrm{C}$. The vortex was initially in gradient wind and hydrostatic balance and located at the center of each mesh. The model was integrated for $192 \mathrm{~h}$ with hourly output except that output at 6-min intervals were saved during $108-155 \mathrm{~h}$ of the simulation for detailed potential temperature budget analysis. Further details of the model setup and simulation can be found in Wang et al. $(2016,2019)$.

The SEF and the ERC in the simulated TC has been investigated deeply in Wang et al. (2019) and can be briefly overviewed from Fig. 1a, which shows the timeradius cross sections of the azimuthal-mean vertical motion at 3-km height and tangential wind speed at 1.5-km height from 72 to $192 \mathrm{~h}$ of the simulation. Prior to the SEF, which is after about $123-126 \mathrm{~h}$ of simulation, strong upward motion occurred in the primary eyewall centered at a radius of around $35 \mathrm{~km}$ from the storm center, with active inner spiral rainbands as inferred from the azimuthal-mean upward motion between radii of $60-80 \mathrm{~km}$. Some scattered upward motions developed at a radius of about $180 \mathrm{~km}$ after $108 \mathrm{~h}$ of simulation and became gradually more organized and propagated radially inward in the azimuthal mean. This indeed reflects the development of a strong outer spiral rainband (not shown) that was initiated in the upper troposphere and then extended downward and propagated radially inward [see Wang et al. (2019) for more detailed discussion]. By around 123-126 h, the secondary eyewall formed between $70-$ and $90-\mathrm{km}$ radii, with weak subsidence in the moat area between the primary and secondary eyewalls.

After its formation, the secondary eyewall intensified and also contracted while the inner eyewall started weakening about $3-6 \mathrm{~h}$ after the SEF as we can see from the strength of upward motion in Fig. 1a. Note that during much of the time period that the inner and outer eyewalls coexisted (viz., between 126 and $144 \mathrm{~h}$ ), the low-level azimuthal-mean maximum tangential wind experienced a considerable weakening as found in a typical ERC discussed in many previous studies based on observations and numerical simulations (e.g., Willoughby et al. 1982; Huang et al. 2012; Bell et al. 2012; Sun et al. 2013; Zhang et al. 2017; Wang et al. 2019). As the outer eyewall further contracted, the inner eyewall continued weakening and eventually dissipated by about $156 \mathrm{~h}$ of the simulation as we can see from the upward motion in Fig. 1a. This means that the doubleeyewall structure was maintained for more than $24 \mathrm{~h}$ in the simulation. Note that the resultant single eyewall showed a larger size than the primary eyewall before the SEF (viz., the eyewall between 50 and $60 \mathrm{~km}$ vs that between 30 and $40 \mathrm{~km}$ ). 
(a) vertical velocity

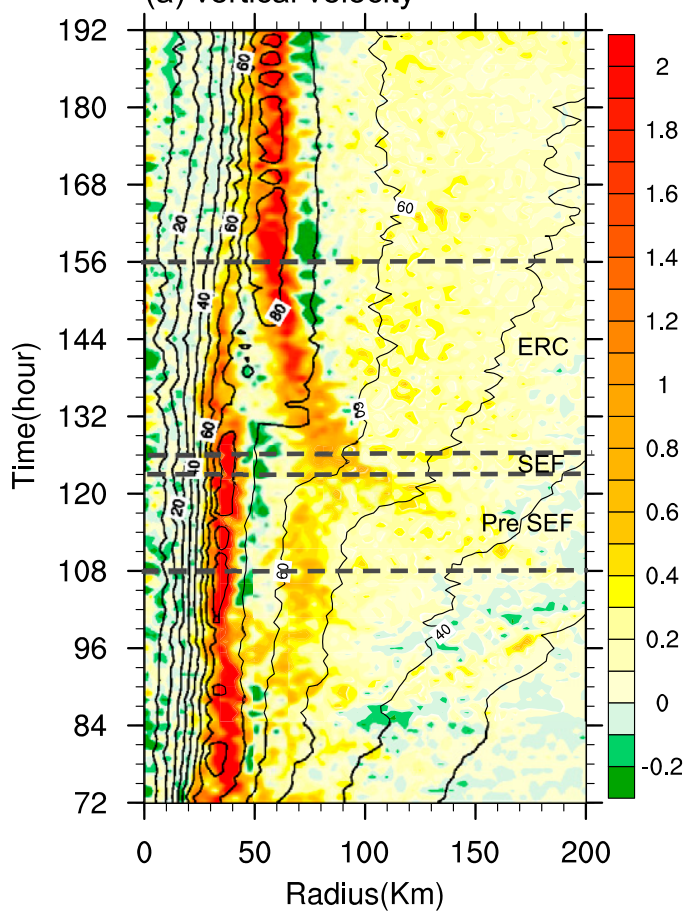

(b) inertial stability

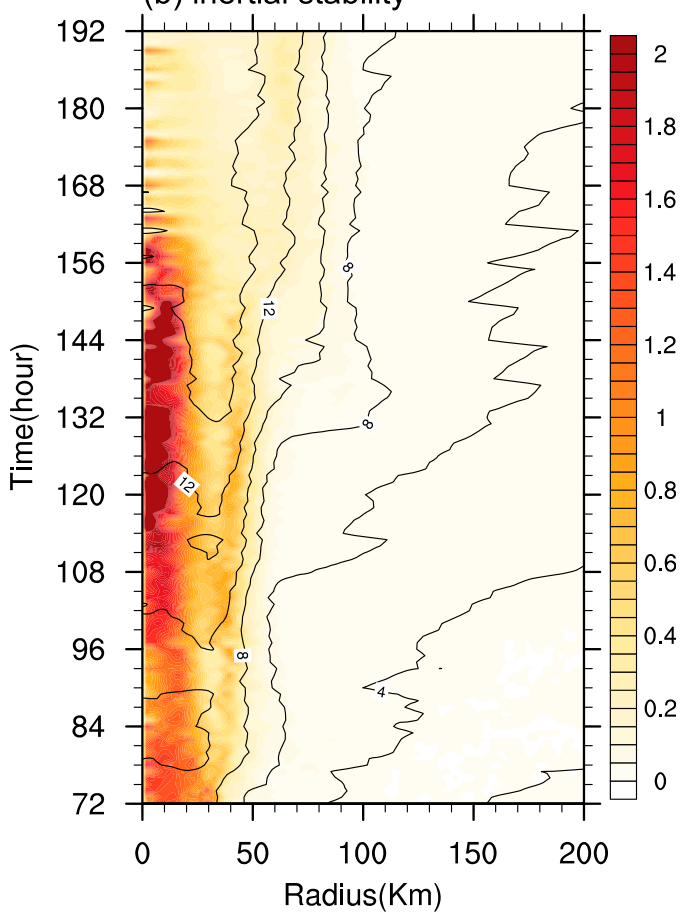

FIG. 1. Time-radius Hovmöller diagrams of (a) the azimuthal-mean vertical motion at $3 \mathrm{~km}$ (color shaded,

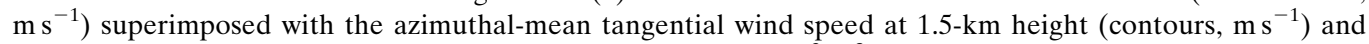
(b) the azimuthal-mean inertial stability (color shaded, $1.0 \times 10^{-3} \mathrm{~s}^{-2}$ ) superimposed with the azimuthal-mean potential temperature anomaly (contours, K) averaged in a layer between 10- and 12-km heights from 72 to $192 \mathrm{~h}$ of simulation. In (a), the dashed horizontal lines mark the three time periods as indicated.

Although the azimuthal-mean tangential wind experienced an overall outward expansion during the simulation, a rapid outward expansion occurred from $108 \mathrm{~h}$ of the simulation (prior to the SEF). This expansion was largely contributed by the development and inward propagation of a strong outer spiral rainband as mentioned above (Wang et al. 2019). A secondary maximum in the azimuthal-mean tangential wind at $1.5-\mathrm{km}$ height appeared at a radius of $75 \mathrm{~km}$ by about $123-126 \mathrm{~h}$ of simulation, consistent with the formation of the secondary eyewall as inferred from the azimuthal-mean vertical motion discussed above. Note that different from the azimuthal-mean vertical motion, the coexistence of two local maxima in the azimuthal-mean tangential wind speed corresponding to the inner and outer eyewalls is less obvious.

To give an overview on the evolution of the vertical structure of the vortex during the SEF and subsequent ERC, we show in Fig. 2 the radius-height cross sections of the azimuthal-mean tangential wind speed and diabatic heating at 6-h intervals from 102 to $168 \mathrm{~h}$ of simulation. These cross sections provide an axisymmetric view of the SEF and the subsequent ERC, in particular for changes in the azimuthal-mean tangential wind in the mid- to upper troposphere. We can see tangential wind expanded outward in response to diabatic heating associated with outer spiral rainbands in the outer radii prior to the SEF (102-120 h in Fig. 2), and the deepening of the azimuthal-mean tangential wind corresponding to the formation and the subsequent intensification of the outer eyewall. Finally, the vertical structure typical of a singleeyewall storm occurred after the dissipation of the inner eyewall by $156 \mathrm{~h}$ of simulation.

\section{Evolution of the warm-core structure}

The warm-core structure is often referred to as the positive temperature or potential temperature anomalies from the unperturbed environment. In this study, the warm core is defined as the positive deviation (perturbation) of the azimuthal-mean potential temperature from the environmental potential temperature. The latter is defined as the mean potential temperature averaged in the annulus between $550-$ and $650-\mathrm{km}$ radii at any given time in the simulation. This definition is broadly consistent with those used in both previous observational and numerical studies (Liu et al. 1997; Knaff et al. 2004; Halverson et al. 2006; Stern and Nolan 2012; 

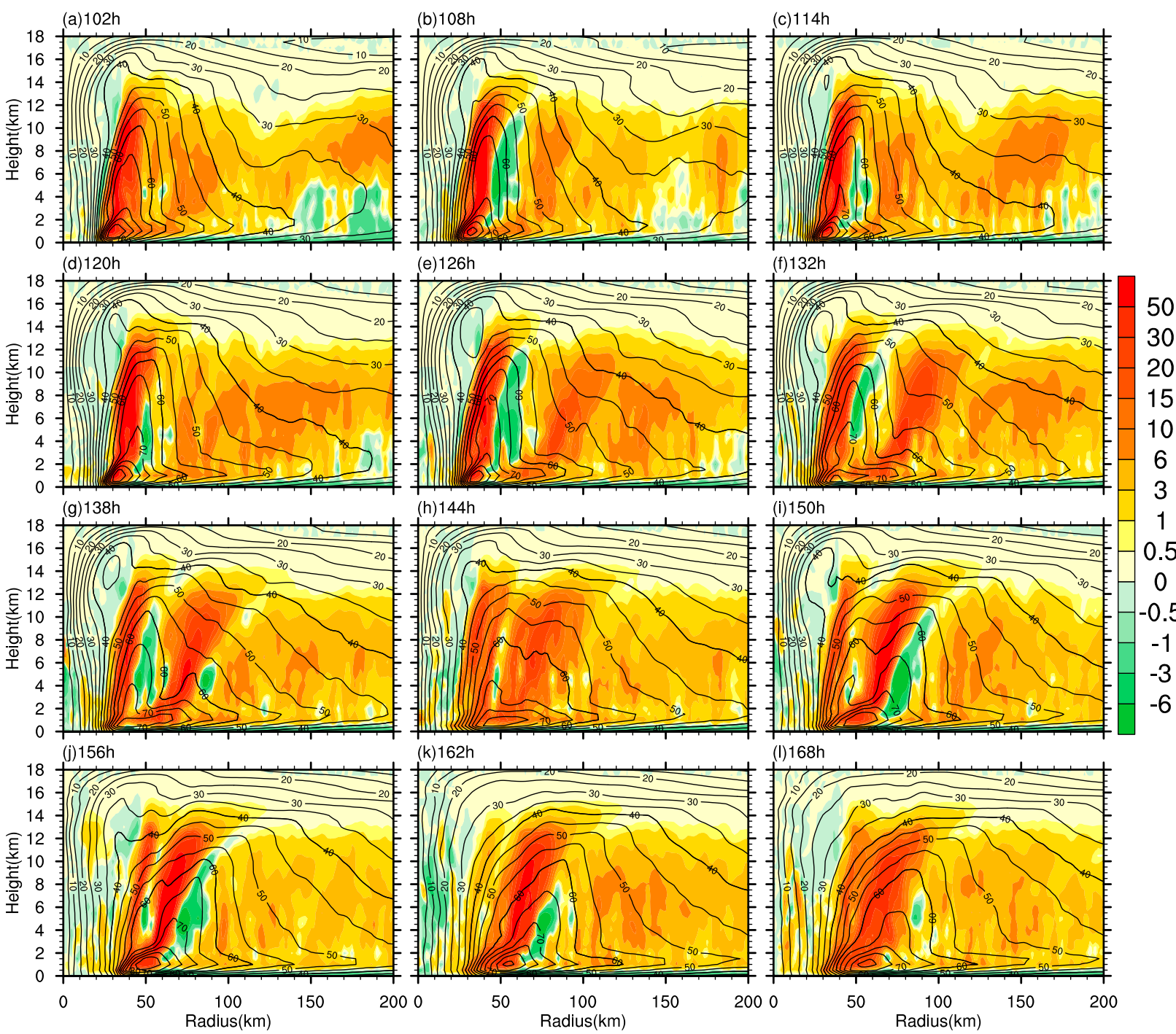

FIG. 2. The instantaneous azimuthal-mean diabatic heating rate (shaded, $\mathrm{K} \mathrm{h}^{-1}$ ) and tangential wind (contour interval of $5 \mathrm{~m} \mathrm{~s}{ }^{-1}$ ) at $6-\mathrm{h}$ intervals from 102 to $168 \mathrm{~h}$ of the simulation, showing the onset of the SEF (around $126 \mathrm{~h}$ ) and the subsequent ERC from 126 through $168 \mathrm{~h}$, followed by the single-eyewall structure afterward.

Stern and Zhang 2013; Ohno and Satoh 2015). Although the definition of the environmental potential temperature seems to be arbitrary, Stern and Nolan (2012) argued that (potential) temperature averaged over an area that is several hundred kilometers away from the storm center is suitable for the study of the warm-core structure. Our definition of the warm core is acceptable since the focus of this study is to examine the evolution and the associated mechanism of the warm-core structure, not the relationship between the eye thermal anomalies with the vortex strength or the height of the warm core, which could be affected by the chosen environmental vertical profile (Durden 2013).

Figure 3 shows the radius-height cross section of the azimuthal-mean perturbation potential temperature at 12-h intervals from 90 to $174 \mathrm{~h}$ of simulation, including the stages prior to and during the SEF and the subsequent ERC period. At $90 \mathrm{~h}$, namely well before the SEF, the storm shows a double warm-core structure, a midlevel warm core with a maximum potential temperature anomaly of $\sim 10-12 \mathrm{~K}$ centered at around $8-\mathrm{km}$ height and an upper-level warm core with a maximum potential temperature anomaly of $\sim 14-16 \mathrm{~K}$ centered at around 15-km height (Fig. 3a). Such a double warmcore structure in TCs has been reported in many previous studies as well (Hawkins and Imbembo 1976; Schwartz et al. 1996; Kieu and Tallapragada 2014; Kieu et al. 2016). However, as the storm continued intensifying slowly, the weak lower warm-core maximum disappeared, while the upper warm core rapidly strengthened and extended upward. By $102 \mathrm{~h}$, the $10-\mathrm{K}$ 

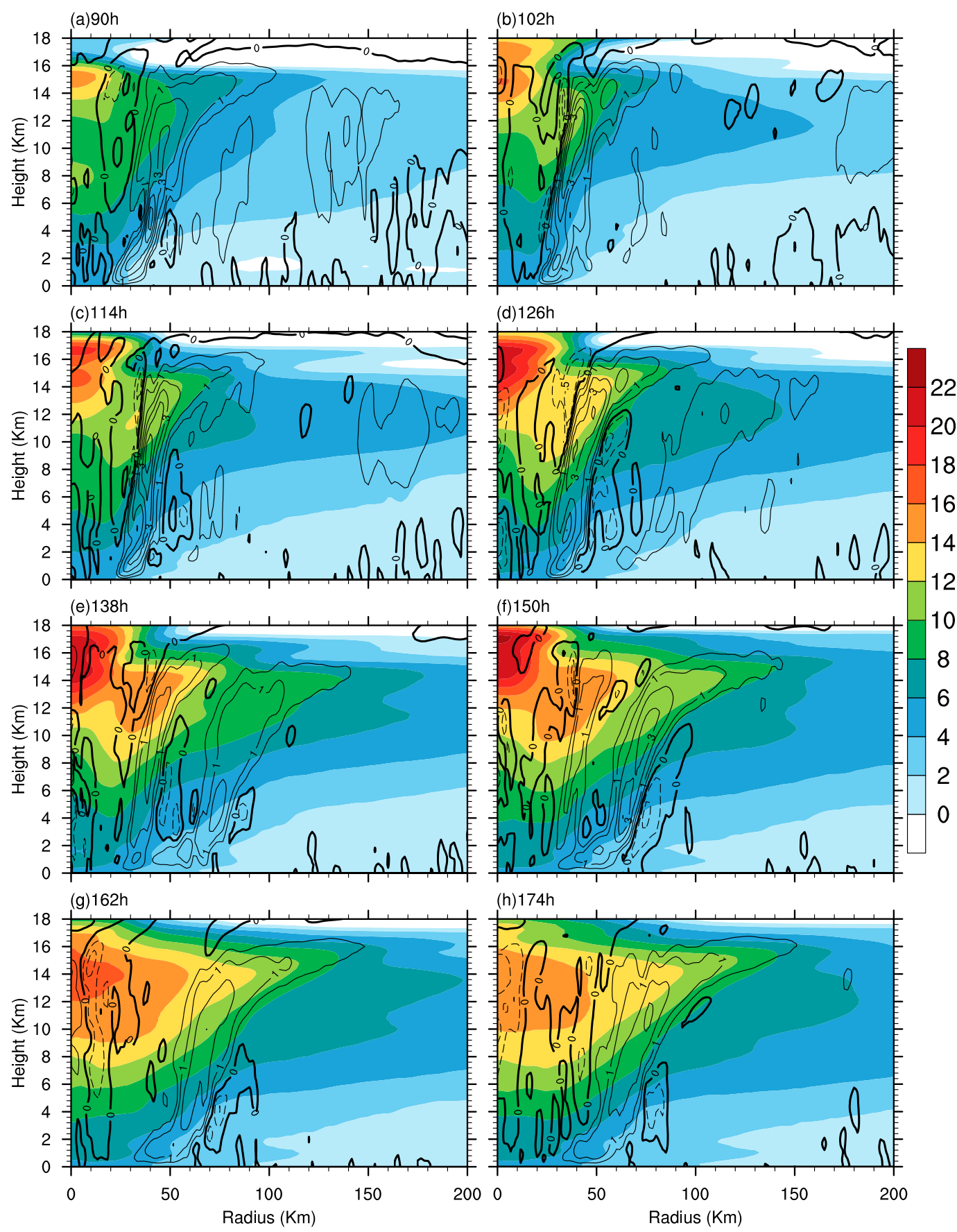

FIG. 3. Radial-vertical cross sections of perturbation potential temperature (color shaded, K) with respect to the environment from 90 to $174 \mathrm{~h}$ at 12 -h intervals overlaid with the corresponding azimuthal-mean vertical velocity contoured at $-0.5,-0.25,0.0$ (thick) $, 0.5,1.0,2.0,3.0,4.0$, and $5.0 \mathrm{~m} \mathrm{~s}^{-1}$. Note that negative contours are dashed, and this applies to subsequent figures as well.

isoline showed an outward and downward expansion to 9-km height (Fig. 3b). This pattern further strengthened, and an off-center warm-ring structure appeared to center at a radius of $30 \mathrm{~km}$ from the storm center and a height of about $11 \mathrm{~km}$ by $114 \mathrm{~h}$ of simulation (Fig. $3 \mathrm{c}$ ), namely, during the period of significant outward expansion of tangential wind (Fig. 2b). This can be more easily seen from the azimuthal-mean inertial stability and potential temperature anomaly averaged between $10-$ and $12-\mathrm{km}$ heights as shown in Fig. 1b. Note that 
the outward expansion of the azimuthal-mean potential temperature anomaly was accompanied with the outward expansion of the azimuthal-mean tangential wind prior to and during the SEF period (Figs. 1a and 1b).

With the progressive inward propagation and downward penetration of the outer rainbands and the formation of the new outer eyewall [see Wang et al. (2019) for more details], the off-center warm ring continued strengthening and expanding both outward and downward (Figs. 3d and 3e). This off-centered warm-ring structure is similar to the establishment of the warm-ring structure related to the single-eyewall TCs proposed by Schubert et al. (2007) and the concentric-eyewall TCs by Rozoff et al. (2008). As the outer eyewall intensified, convection in the inner eyewall experienced a continuous weakening (Figs. 1a and 2). However, the upperlevel warm core associated with the original inner eyewall maintained its strength for a relatively long period while the lower off-center warm ring continued strengthening and eventually merged with the warm core of the original primary eyewall by $174 \mathrm{~h}$ after the inner eyewall disappeared (Figs. $3 g$ and $3 h$ ). Note that different from the dissipation of convection in the inner eyewall around $156 \mathrm{~h}$ (Figs. 1 and 2), the lower off-center warm ring merged with the central upper warm core at the end of the ERC (Figs. 3g and 3h), showing a large warm-core structure after the ERC, similar to the warmcore structure in a typical single-eyewall TC.

To see more clearly how the warm-core structure evolved in the simulated storm, we show in Fig. 4 the radius-height cross sections of the 12-h change in the azimuthal-mean perturbation potential temperature overlaid with the corresponding 12-h-averaged azimuthal-mean vertical velocity, based on the hourly model outputs from 102 to $174 \mathrm{~h}$ of the simulation. Note that the azimuthal-mean vertical velocity indicates the location of eyewall ascent during the SEF and the subsequent ERC. We can see from Fig. 4 that prior to the SEF the large warming occurred in the upper-eye region and an off-center warming appeared mainly in the upper part of the outwardly tilted eyewall and in the outflow layer from 102 to $114 \mathrm{~h}$ (Fig. 4a). There is a thin layer of cooling in the lower stratosphere outside the eye region above $17-\mathrm{km}$ height. For the next 12-h period (114-126 h; Fig. 4b), namely during the onset stage of the SEF, the overall changes in the azimuthal-mean potential temperature are similar to the previous $12 \mathrm{~h}$ except for the distribution of cooling in the lower stratosphere. The completion of the SEF yielded substantial warming in the next $12 \mathrm{~h}$ between 126 and $138 \mathrm{~h}$ (Fig. 4c), in particular near the inner edge of the inner eyewall in the upper troposphere and in the moat area between the two eyewalls in the mid- to upper troposphere, and in the outflow layers of the two eyewalls.
A small cooling appeared at the top of the eye region above $16-\mathrm{km}$ height by $138-150 \mathrm{~h}$ (Fig. $4 \mathrm{~d}$ ). This upperlevel cooling in the eye region strengthened and extended downward to $14-\mathrm{km}$ height by $150-162 \mathrm{~h}$ (Fig. 4e). This cooling reflected the weakening of the inner eyewall and the strengthening and contracting of the outer eyewall. Well after the completion of the SEF, the inner eyewall experienced a continuous weakening as inferred from the azimuthal-mean vertical motion in Figs. $4 \mathrm{~d}$ and $4 \mathrm{e}$. As a result, the warming near the inner edge of the inner eyewall in the upper troposphere largely weakened. Furthermore, with the maturing of the outer eyewall, the warming in both the moat area and the outflow layer of the outer eyewall also showed a weakening (Figs. $4 \mathrm{~d}$ and $4 \mathrm{e}$ ). Note that a large cooling appeared in the eye above $14 \mathrm{~km}$ and a warming appeared in the eye immediately below between 6- and $13-\mathrm{km}$ heights as the inner eyewall decayed, indicating a lowering warm-core structure and the transition from the double-eyewall structure to a single-eyewall structure with a large size eye and a greatly sloped eyewall (Fig. 4e). Finally, after the completion of the ERC (162-174h; Fig. 4f), the potential temperature tendency became relatively small, indicating the quasi-steady-state evolution of the simulated storm.

A comparison between Figs. 3 and 4 indicates that the off-center warm-ring structure prior to and during the SEF and subsequent ERC is largely related to the warming first near the inner edge of the primary eyewall and later partly in the moat area. The outward extension of the overall warm-core structure is related to the warming in the moat area and in the outflow layer, consistent with the enlarged inner core of the storm with the ERC (Figs. 1 and 2). Note that the lower warm-core structure after the completion of the ERC compared to the pre-SEF structure reflects the large eyewall size and more outwardly tilted eyewall structure as inferred from the balanced dynamics (Wang 2008). Stern and Zhang (2016) argued that the lower height of the warm-core maximum in a simulation of Hurricane Earl (2010) reflected a tendency of the too-great eyewall slope in the simulation than in observations.

\section{Potential temperature budget analysis}

To understand the predominant processes that controlled the evolution of the warm-core structure of the simulated storm prior to SEF, during SEF, and during the subsequent ERC, we performed an azimuthal mean potential temperature budget analysis. The budget equation for the azimuthal-mean potential temperature $\bar{\theta}$ can be written as 

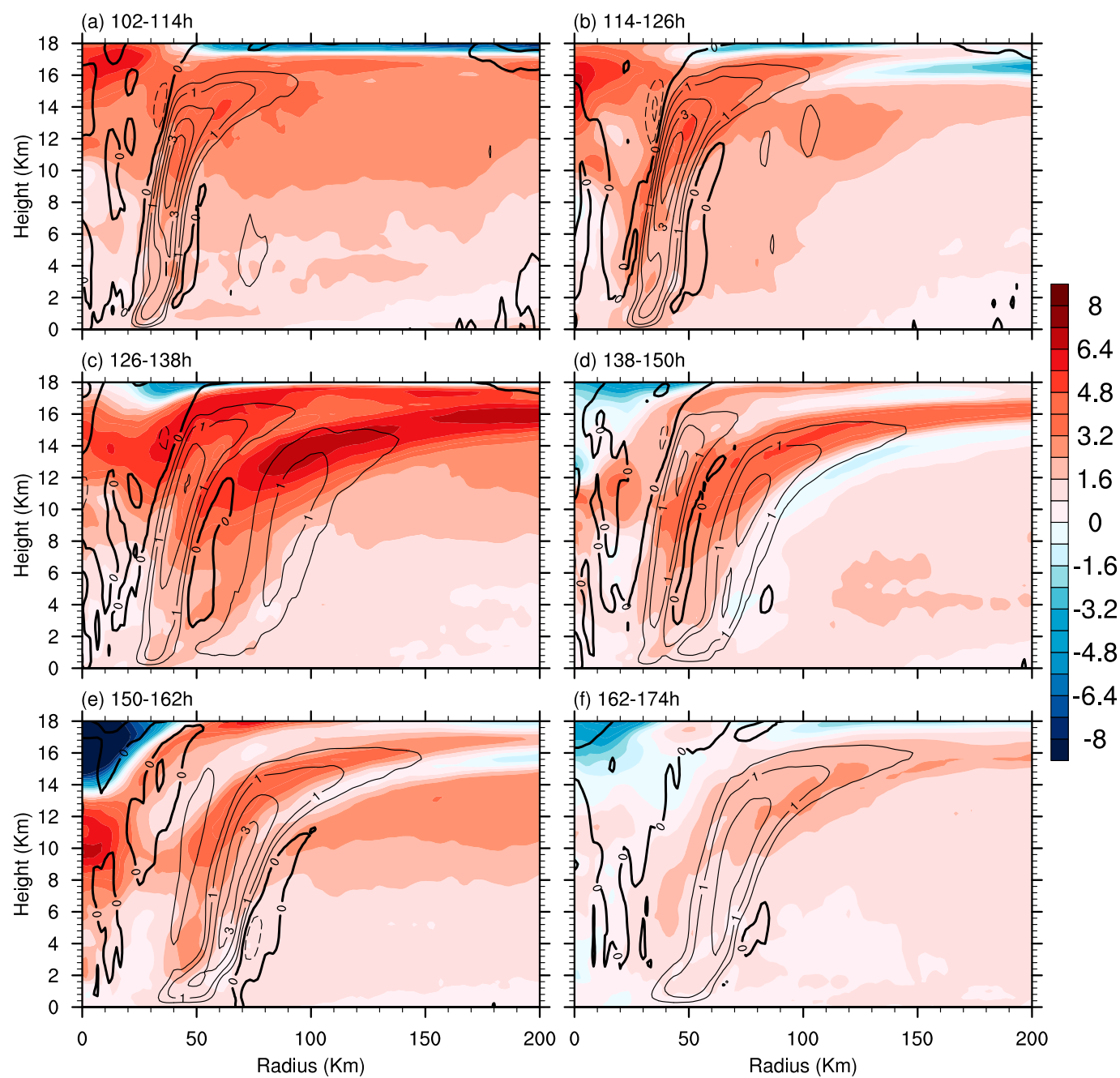

FIG. 4. Radial-vertical cross sections of 12-h changes in the azimuthal-mean potential temperature (color shaded, K) overlaid with the 12 -h-averaged azimuthal-mean vertical velocity contoured at $-0.5,-0.25,0.0$ (thick), 0.5, 1.0, 2.0, 3.0, and $4.0 \mathrm{~m} \mathrm{~s}^{-1}$.

$$
\frac{\partial \bar{\theta}}{\partial t}=\mathrm{TADV}+\mathrm{HEAT}+\mathrm{RAD}+\mathrm{PBL}+\mathrm{DIFF}
$$

where TADV is the azimuthal-mean tendency due to total advection of potential temperature (including both azimuthal-mean and eddy components); HEAT is the contribution by the azimuthal-mean diabatic heating due to phase changes from cloud microphysics; and RAD, PBL, and DIFF are the contributions by longwave and shortwave radiative forcing, boundary layer turbulent mixing (including surface sensible heat flux), and subgrid-scale horizontal diffusion, respectively. To minimize the errors in the budget, all terms are directly output at every 6 min during the model simulation, and Eq. (1) is then numerically integrated in time, as done by Stern and Zhang (2013).

Three time periods were chosen for the budget analysis, including the stages prior to the SEF (110-116h), shortly after the completion of the SEF (125-131 h), and near the end of the ERC (150-156h). Figure 5 shows the radius-height cross sections of the 6-h changes in the azimuthal-mean potential temperature obtained directly from the model outputs and that obtained as the sum of all terms on the right-hand side of Eq. (1) based on the model outputs at 6-min intervals during the three given time periods, respectively. We can see that overall, both the direct changes and the changes obtained based on Eq. (1) agree qualitatively and quantitatively well. Some discrepancies exist near the vertical axis of the storm center, which are mainly due to the interpolation errors, also to the errors induced by using model outputs at every $6 \mathrm{~min}$, and not at every time step. Nevertheless, the changes based on Eq. (1) compare well with the actual potential temperature changes, especially in the region of the SEF for the three given time periods, 

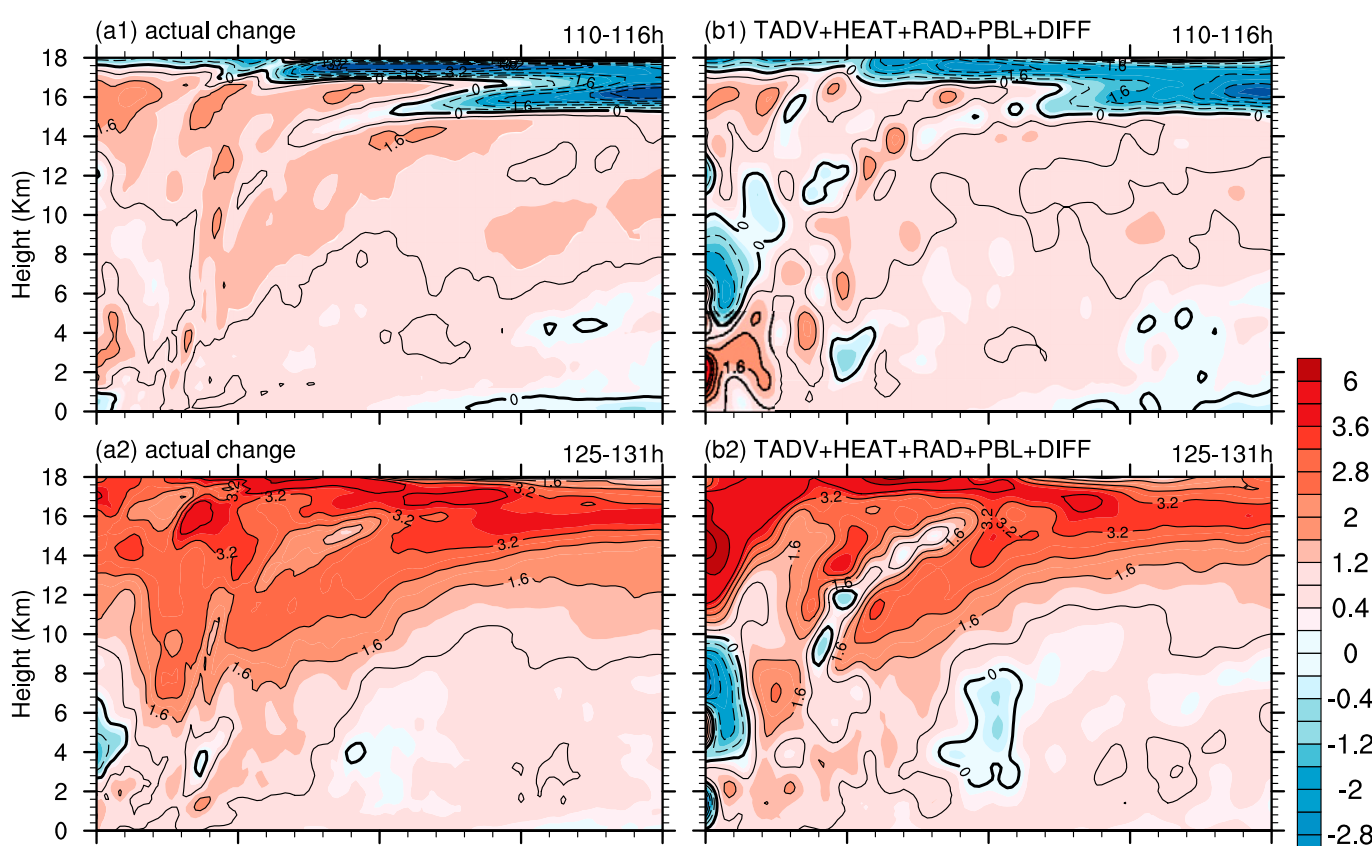

3.6
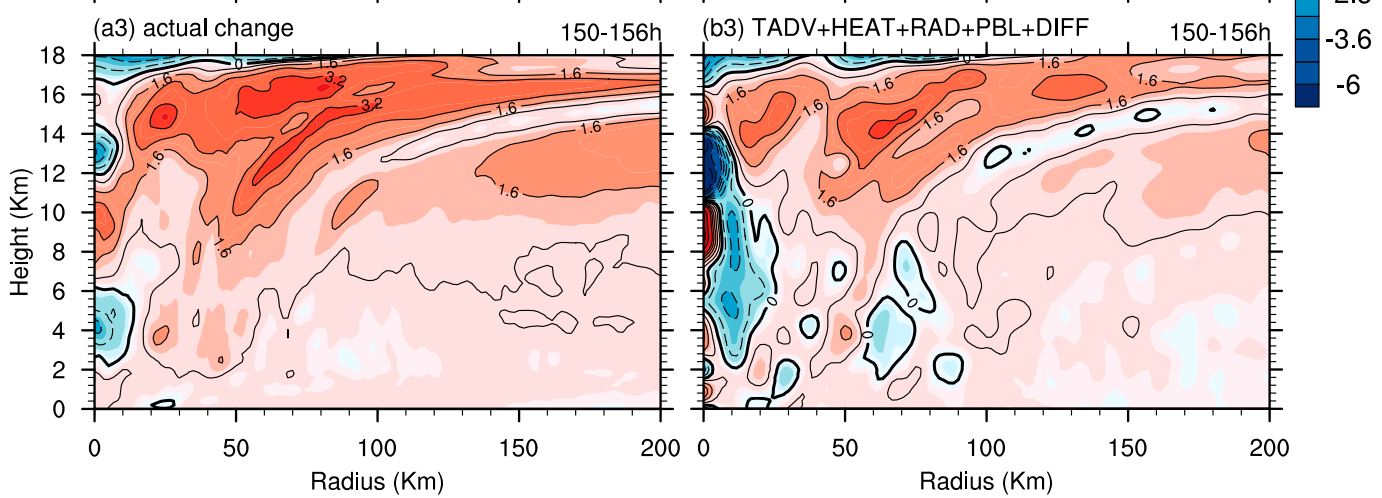

FIG. 5. Radial-vertical cross sections of (a) the actual 6-h changes of potential temperature and (b) those from the budget using Eq. (1) based on the model output at 6-min intervals (color shaded, K). Contours shown are $-8,-6$, $-4,-3.2,-2.4,-1.6,-0.8,0$ (thick), $0.8,1.6,2.4,3.2,4,6$, and $8 \mathrm{~K}$ for the three time periods of hours (top) $110-116$, (middle) 125-131, and (bottom) 150-156.

suggesting that the budget analysis based on Eq. (1) can provide insights into the relative importance of processes that contributed to the warming discussed in section 3.

Figure 6 shows the radius-height cross sections of the azimuthal-mean TADV, diabatic heating rate (HEAT), and the sum of three small terms on the rhs of Eq. (1), all averaged in the corresponding three given time periods, respectively. Note that the corresponding diagnosed potential temperature tendencies (the 6-h changes shown in the right column in Fig. 5) are shown in contours as a background to illustrate the relative contribution of TADV, HEAT, and the sum of the last three terms on the rhs of Eq. (1) to the total budgeted potential temperature tendency. We can see that the net contribution to the potential temperature budget by the last three terms in all three periods is rather small [Figs. 6c(1)-(3)]. The warming tendencies in the lower part and cooling tendencies in the upper part in the boundary layer are mainly contributed by PBL, the cooling tendencies in the upper troposphere above 14-km height outside the eye is mainly contributed by RAD, and overall contributions by DIFF is quite small throughout the troposphere. Therefore, these three terms do not contribute systematically to the warming tendency of the warm-core evolution during the SEF and ERC and thus are not included in our following discussions. Both TADV and HEAT are leading terms but have opposite sign in all three stages (Figs. 6a and $6 \mathrm{~b})$. Overall, the sum of the two terms gives a net tendency that is very similar to the 6 -h potential temperature changes during all the three periods (right column in 

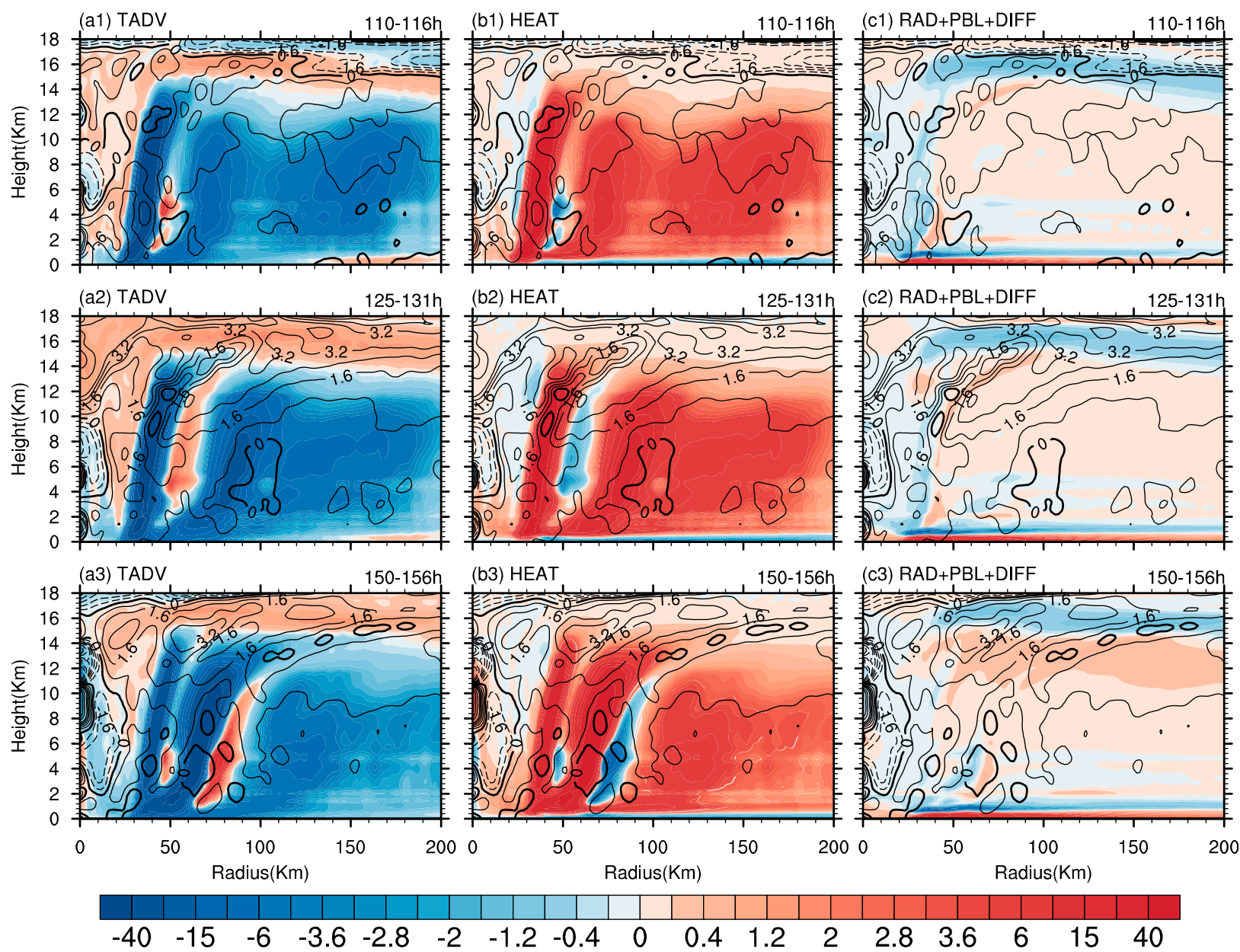

FIG. 6. Radial-vertical cross sections of the 6-h azimuthal-mean potential temperature change from the budget as the sum of all terms on the rhs of Eq. (1) [contour contoured at $-8,-6,-4,-3.2,-2.4,-1.6,-0.8,0$ (thick), 0.8, 1.6, 2.4, 3.2, 4, 6, and $8 \mathrm{~K}$ ], the contributions by (a) total advection term (TADV, color shaded, K), (b) diabatic heating rate (HEAT, color shaded, K), and (c) the sum of RAD, PBL, and DIFF (color shaded, K) for three time periods of hours (top) 110-116, (middle) 125-131, and (bottom) 150-156, based on model outputs at 6-min intervals.

Fig. 5). This means that the evolution of the warm-core structure in the simulated storm is mainly controlled by both advection and diabatic heating processes, regardless the stage of the ERC. Note that although contributions by diabatic heating and advection largely offset each other, it is their residual that contributes to the potential temperature changes in the simulation. We can see that overall the advection contributes dominantly to the warming tendency in both the eye region and in the outflow layer above about 14-km height and diabatic heating contributes dominantly to the warming tendency in the eyewall region, in particular in the upper part of the eyewall convection.

Since the SEF in the simulation is mainly triggered by asymmetric processes related to the activity of outer spiral rainbands (as discussed in Wang et al. 2019), it is interesting to see the relative contributions to TADV by the azimuthal-mean advection and the asymmetric eddy processes. Following Stern and Zhang (2013) and Ohno and Satoh (2015), we further split the total advection term (TADV) into the azimuthal-mean component and the asymmetric (eddy) component. Namely, the TADV can be rewritten below:

$$
\mathrm{TADV}=-\bar{u} \frac{\partial \bar{\theta}}{\partial r}-\bar{w} \frac{\overline{\partial \theta}}{\partial z}-\overline{u^{\prime} \frac{\partial \theta^{\prime}}{\partial r}}-\overline{w^{\prime} \frac{\partial \theta^{\prime}}{\partial z}}
$$

where $u$ and $w$ are radial and vertical velocity, $\theta$ is potential temperature, and $r$ and $z$ are radius and height. Overbars denote the azimuthal mean and primes denote the deviation from the corresponding azimuthal mean. The terms on the rhs of Eq. (2) are, respectively, the mean radial advection (MRADV), mean vertical advection 

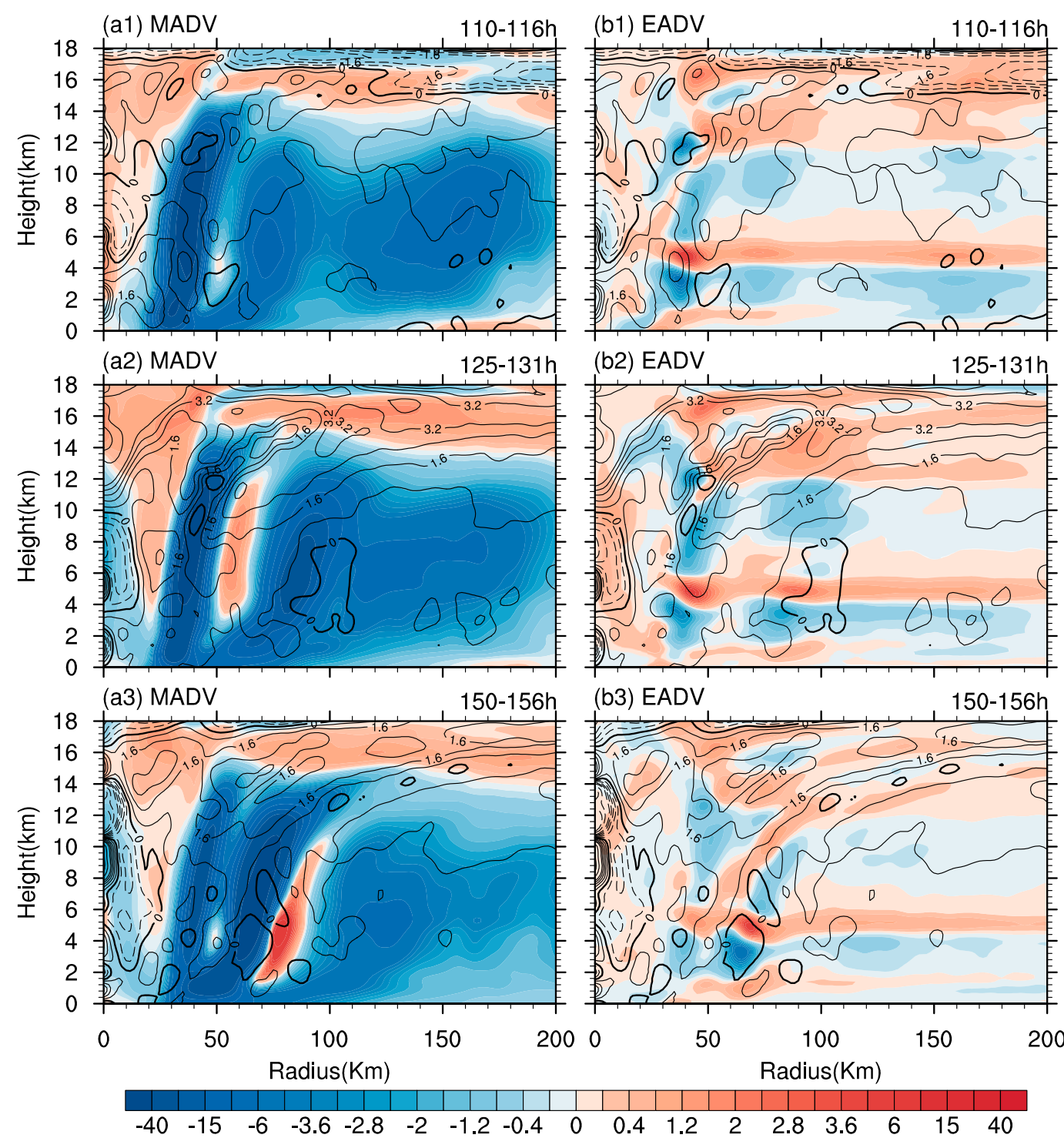

FIG. 7. Radial-vertical cross sections of the 6-h azimuthal-mean potential temperature change from the budget as the sum of all terms on the rhs of Eq. (1) [contoured at $-8,-6,-4,-3.2,-2.4,-1.6,-0.8,0$ (thick), 0.8, 1.62.4, 3.2, 4,6 , and $8 \mathrm{~K}$ ], and contributions by (a) the azimuthal-mean advection (MADV, color shaded, K) and (b) the eddy advection (EADV, color shaded, K) for three time periods of hours (top) 110-116, (middle) 125-131, and (bottom) $150-156$, based on model outputs at 6-min intervals.

(MVADV), eddy radial advection (ERADV), and eddy vertical advection (EVADV).

Figure 7 compares the total azimuthal-mean advection term (MADV; the sum of MRADV and MVADV) and the total eddy advection term (EADV; the sum of ERADV and EVADV) in the same three periods as shown in Fig. 6. Note that the sum of the mean and eddy advection terms is consistent with the corresponding TADV shown in the left column in Fig. 6. As we can see from Fig. 7, although the EADV is relatively small in all three periods, it still played some role in the overall warming above $12-\mathrm{km}$ height in the upper troposphere.
The eddy warming in a thin layer near the $5-\mathrm{km}$ height is associated with the freezing layer and is largely contributed by eddy vertical advection (not shown). Nevertheless, the result indicates that although the eddy processes contribute somewhat to the upper-level warming, the mean advection is still the dominant process in causing the warming pattern and evolution in the simulated storm.

Prior to the SEF, the overall warming is relatively small and mainly located in the eye region in the upper troposphere, in the outwardly tilted primary eyewall, and in a shallow layer corresponding to the outflow of 

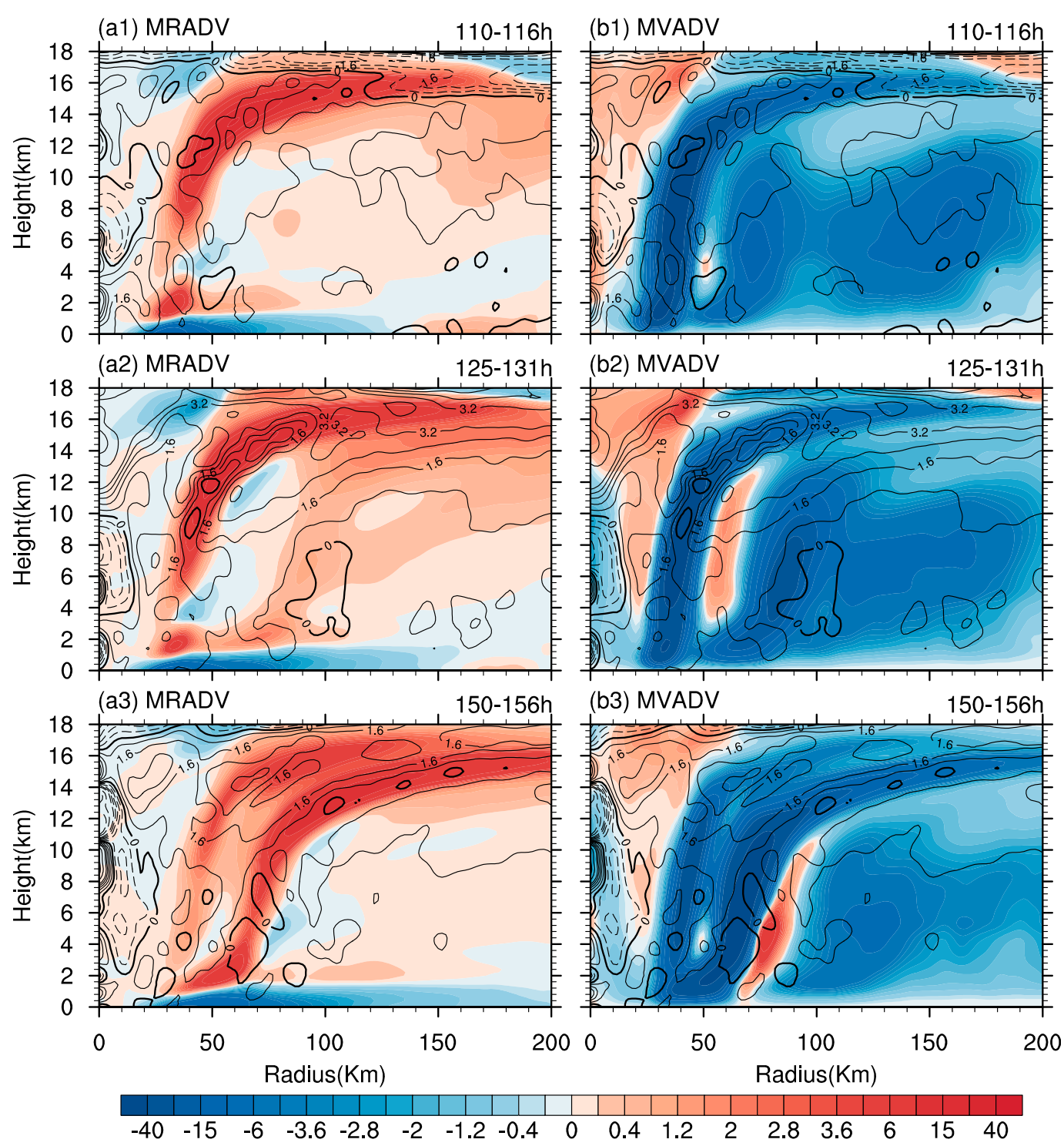

FIG. 8. The radial-vertical cross sections of the 6-h azimuthal-mean potential temperature change from the budget as the sum of all terms on the rhs of Eq. (1) [contoured at $-8,-6,-4,-3.2,-2.4,-1.6,-0.8,0$ (thick), $0.8,1.6,2.4,3.2,4,6$, and $8 \mathrm{~K}$ ], and contributions by (a) the azimuthal-mean radial advection (MRADV, color shaded, K) and (b) the azimuthal-mean vertical advection (MVADV, color shaded, K) for three time periods of hours (top) 110-116, (middle) 125-131, and (bottom) 150-156, based on model outputs at 6-min intervals.

the simulated storm [Figs. 5a(1) and 5a(2)]. The warming in the outflow layer above $14-\mathrm{km}$ height is dominated by TADV [Fig. 6a(1)], which is mainly the mean radial horizontal advection induced by outflow [MRAD; Fig. 8a(1)], whereas the warming just below 14-km height is mainly associated with diabatic heating [Fig. 6b(1)]. The warming in the eye region is predominantly contributed by TADV, which is mainly due to subsidence warming included in mean vertical advection [MVAD; Fig. 8b(1)]. During this budget period, the shallow cooling region above $16-\mathrm{km}$ height outside of a radius of about $50 \mathrm{~km}$ resulted from TADV, which is mainly related to the mean vertical advection [Fig. 8b(1)].

With the completion of the SEF $[125-131 \mathrm{~h}$ of the simulation; Figs. $5 \mathrm{~b}(1)$ and $5 \mathrm{~b}(2)]$, large warming in the upper troposphere above 14-km height outside the eyewall is contributed by TADV, which is dominated by mean radial advection in the outflow layer [Fig. 8a(2)], and the warming in the upper-level eye region is contributed solely by TADV because of subsidence warming [Fig. 8b(2)]. The warming in the primary eyewall and in the upper part of the newly formed secondary eyewall is largely contributed by diabatic heating [Fig. 6b(2)]. 
The warming within the moat area (between the two eyewalls) is largely contributed by subsidence warming [Figs. $6 a(2)$ and $8 b(2)$ ]. The result demonstrates that although contributions by diabatic heating and adiabatic cooling due to vertical advection largely offset each other in the convective (eyewall) regions, some warming still occurred within the inner-core region, in particular in the upper part of the eyewall. Note that both the subsidence warming near the inner edge of the primary eyewall [Fig. $8 \mathrm{~b}(2)]$ and the warming in the primary eyewall due to diabatic heating [Fig. 6b(2)] are responsible for the formation of the off-center warmring structure (Figs. 3b-f).

Near the end of the eyewall replacement $[150-156 \mathrm{~h}$; Fig. 6a(3)], the upper-level warming due to TADV shrank radially inward and also weakened, consistent with the weakening of the inner eyewall and the contraction of the outer eyewall. Nevertheless, TADV is the only term that causes the upper-level (above $14 \mathrm{~km}$ ) warming both in the eye region and in the upper-level outflow layer, while below about 14-km height diabatic heating accounts for the warming in the upper part of the weakening inner eyewall and in the strengthening outer eyewall. Nevertheless, the warming trend decreased with time (Figs. 4e and 4f) and eventually a quasi-steady large single-eyewall storm with a relatively large and lower warm-core structure emerged (Figs. 3g and $3 h$ ).

The above potential temperature budget analysis demonstrates that the warming in the eye region is solely contributed by subsidence warming; the upper-level warming outside the eyewall is mostly contributed by the mean radial warm advection of potential temperature with some eddy contribution; and the off-center warming along the inner edge of the eyewall and the warming in the moat area are largely contributed by subsidence. The warming below $14-\mathrm{km}$ in the inner and outer eyewalls is contributed by diabatic heating because part of the diabatic heating could not be offset by advective cooling in the eyewalls, especially in the upper part of the eyewalls.

\section{Conclusions}

Although the SEF in a TC has been studied extensively in recent years, less attention has been given to the evolution of the warm-core structure during the ERC of a TC. In this study, the evolution of the warmcore structure during the SEF and the subsequent ERC in the numerically simulated TC under idealized conditions on an $f$ plane has been examined and understood based on a budget analysis of the azimuthal-mean potential temperature. It was shown that prior to the SEF, the TC exhibited double warm-core structure, respectively, centered in the middle and upper troposphere in the eye region. As the storm intensified with a rapid outward expansion of the azimuthal-mean tangential wind, the warm core strengthened and an off-center warm ring developed near the inner edge of the primary eyewall between 8 - and 16-km heights. During and shortly after the SEF, the upper-level warm core continued strengthening while the off-center warm ring rapidly strengthened. As the secondary eyewall intensified and contracted, and the primary eyewall gradually weakened, the off-center warm ring extended inward and eventually merged with the upper-level inner warm core to form a warm-core structure typical of a singleeyewall TC that has a large eye size with a large outward eyewall slope.

Results from the azimuthal-mean potential temperature budget analysis indicate that the warming in the eye was due to subsidence warming and the warming outside the eye region above 14-km height was largely contributed by radial outward advection of high potential temperature of the warm core. The warming associated with the off-center warm ring during the SEF was largely contributed by subsidence warming near the inner edge of the primary eyewall. The warming associated with the off-center warm ring below 14-km height in the upper part of the primary eyewall was mostly contributed by diabatic heating in the primary eyewall, where the adiabatic cooling due to advection could not totally offset the diabatic heating.

Further analysis indicates that although the azimuthalmean processes dominated the warming, eddy processes, mainly the eddy radial potential temperature advection, also played some role in the warming above 12-km height in the upper troposphere. This is in sharp contrast to the budget analysis for the azimuthal-mean tangential wind discussed in Wang et al. (2019), who showed that in addition to the azimuthal-mean processes, the eddy processes played some important role in spinning up the azimuthal-mean tangential winds in the boundary layer. This is mainly due to the fact that the warm-core structure is a mid- to upper-tropospheric phenomenon.

Results from this study suggest that in addition to the outward expansion of tangential wind, the continuous off-center warming can also serve as an indication of possible onset of the SEF in a strong TC. We also found that the relatively large warming occurred immediately after the SEF in the simulated TC (Fig. 4c), which is likely due to the sharp increase in inertial stability in the primary eyewall region in the mid- to upper troposphere (not shown) and thus the increased efficiency of diabatic heating in producing the in situ warming, as previously 
hypothesized by Rozoff et al. (2008). Note that although this study provides an initial assessment on the evolution of the warm-core structure in a numerically simulated TC with the SEF and the ERC, more simulations could be done to verify the findings from this single simulation in future studies.

Acknowledgments. This study has been supported in part by the National Key R\&D Program of China under Grant 2017YFC1501602, the National Basic Research and Development Project (973 Program) of China under Contract 2015CB452805, and in part by the National Natural Science Foundation of China under Grants 41675044 and 41730960, NSF Grants AGS-1326524 and AGS-1834300, Special Fund for Meteorological Scientific Research in the Public Interest (Grant GYHY201406006), and Basic Research Fund of CAMS $2016 Z 003$ and 2017Y013.

\section{REFERENCES}

Abarca, S. F., and K. L. Corbosiero, 2011: Secondary eyewall formation in WRF simulations of Hurricanes Rita and Katrina (2005). Geophys. Res. Lett., 38, L07802, https://doi.org/10.1029/ 2011 GL047015.

— , and M. T. Montgomery, 2013: Essential dynamics of secondary eyewall formation. J. Atmos. Sci. 70, 3216-3230, https://doi.org/ 10.1175/JAS-D-12-0318.1.

$\longrightarrow$, and — 2014: Departures from axisymmetric balance dynamics during secondary eyewall formation. J. Atmos. Sci., 71, 3723-3738, https://doi.org/10.1175/JAS-D-14-0018.1.

Bell, M. M., M. T. Montgomery, and W.-C. Lee, 2012: An axisymmetric view of eyewall evolution in Hurricane Rita (2005). J. Atmos. Sci., 69, 2414-2432, https://doi.org/10.1175/JAS-D-11-0167.1.

Chen, H., and D.-L. Zhang, 2013: On the rapid intensification of Hurricane Wilma (2005). Part II: Convective bursts and the upper-level warm core. J. Atmos. Sci., 70, 146-172, https:// doi.org/10.1175/JAS-D-12-062.1.

Dai, Y., S. J. Majumdar, and D. S. Nolan, 2017: Secondary eyewall formation in tropical cyclones by outflow-jet interaction. J. Atmos. Sci., 74, 1941-1958, https://doi.org/10.1175/JAS-D16-0322.1.

Didlake, A. C., Jr., P. D. Reasor, R. F. Rogers, and W.-C. Lee, 2018: Dynamics of the transition from spiral rainbands to a secondary eyewall in Hurricane Earl (2010). J. Atmos. Sci., 75, 2909-2929, https://doi.org/10.1175/JAS-D-17-0348.1.

Dudhia, J., 1989: Numerical study of convection observed during the Winter Monsoon Experiment using a mesoscale two-dimensional model. J. Atmos. Sci., 46, 3077-3107, https://doi.org/10.1175/ 1520-0469(1989)046<3077:NSOCOD > 2.0.CO;2.

Durden, S. L., 2013: Observed tropical cyclone eye thermal anomaly profiles extending above $300 \mathrm{hPa}$. Mon. Wea. Rev., 141, 4256-4268, https://doi.org/10.1175/MWR-D-13-00021.1.

Eliassen, A., 1951: Slow thermally or frictionally controlled meridional circulation in a circular vortex. Astrophys. Nor., 5, $19-60$.

Gray, W. M., E. Ruprecht, and R. Phelps, 1975: Relative humidity in tropical weather systems. Mon. Wea. Rev., 103, 685-690, https:// doi.org/10.1175/1520-0493(1975)103<0685:RHITWS>2.0.CO;2.
Hack, J. J., and W. H. Schubert, 1986: Nonlinear response of atmospheric vortices to heating by organized cumulus convection. J. Atmos. Sci., 43, 1559-1573, https://doi.org/10.1175/ 1520-0469(1986)043<1559:NROAVT>2.0.CO;2.

Halverson, J. B., J. Simpson, G. Heymsfield, H. Pierce, T. Hock, and L. Ritchie, 2006: Warm core structure of Hurricane Erin diagnosed from high altitude dropsondes during CAMEX-4. J. Atmos. Sci., 63, 309-324, https://doi.org/10.1175/JAS3596.1.

Hawkins, H. F., and S. M. Imbembo, 1976: The structure of a small, intense hurricane-Inez 1966. Mon. Wea. Rev., 104, 418-442, https://doi.org/10.1175/1520-0493(1976)104<0418:TSOASI> 2.0.CO;2.

Hong, S.-Y., and J.-O. J. Lim, 2006: The WRF single-moment 6-class microphysics scheme (WSM6). J. Korean Meteor. Soc., 42, 129-151.

—, Y. Noh, and J. Dudhia, 2006: A new vertical diffusion package with an explicit treatment of entrainment processes. Mon. Wea. Rev., 134, 2318-2341, https://doi.org/10.1175/ MWR3199.1.

Houze, R. A., Jr., S. S. Chen, B. F. Smull, W. C. Lee, and M. M. Bell, 2007: Hurricane intensity and eyewall replacement. Science, 315, 1235-1239, https://doi.org/10.1126/science.1135650.

Huang, Y.-H., M. T. Montgomery, and C.-C. Wu, 2012: Concentric eyewall formation in Typhoon Sinlaku (2008). Part II: Axisymmetric dynamical processes. J. Atmos. Sci., 69, 662-674, https://doi.org/10.1175/JAS-D-11-0114.1.

Judt, F., and S. S. Chen, 2010: Convectively generated potential vorticity in rainbands and formation of the secondary eyewall in Hurricane Rita of 2005. J. Atmos. Sci., 67, 3581-3599, https://doi.org/10.1175/2010JAS3471.1.

Kain, J. S., and J. M. Fritsch, 1993: Convective parameterization for mesoscale models: the Kain-Fritsch scheme. The Representation of Cumulus Convection in Numerical Models, Meteor. Monogr. No. 46, Amer. Meteor. Soc., 165-177.

Kepert, J. D., 2013: How does the boundary layer contribute to eyewall replacement cycles in axisymmetric tropical cyclones? J. Atmos. Sci., 70, 2808-2830, https://doi.org/10.1175/JAS-D13-046.1.

Kieu, C., and V. Tallapragada, 2014: On the development of double warm cores in intense tropical cyclones in the HWRF Model. 31st Conf. on Hurricanes and Tropical Meteorology, San Diego, CA, Amer. Meteor. Soc., 17D.4. https://ams.confex.com/ams/ 31Hurr/webprogram/Paper243675.html.

,$--1-$ D.-L. Zhang, and Z. Moon, 2016: On the development of double warm-core structures in intense tropical cyclones. J. Atmos. Sci., 73, 4487-4506, https://doi.org/10.1175/JAS-D16-0015.1.

Knaff, J. A., S. A. Seseske, M. DeMaria, and J. L. Demuth, 2004: On the influences of vertical wind shear on symmetric tropical cyclone structure derived from AMSU. Mon. Wea. Rev., 132, 2503-2510, https://doi.org/10.1175/1520-0493(2004)132<2503: OTIOVW $>2.0 . \mathrm{CO} ; 2$.

Komaromi, W. A., and J. D. Doyle, 2017: Tropical cyclone outflow and warm core structure as revealed by HS3 dropsonde data. Mon. Wea. Rev., 145, 1339-1359, https://doi.org/10.1175/ MWR-D-16-0172.1.

Kossin, J. P., and M. D. Eastin, 2001: Two distinct regimes in the kinematic and thermodynamic structure of the hurricane eye and eyewall. J. Atmos. Sci., 58, 1079-1090, https://doi.org/ 10.1175/1520-0469(2001)058<1079:TDRITK > 2.0.CO;2.

Kurihara, Y., 1975: Budget analysis of a tropical cyclone simulated in an axisymmetric numerical model. J. Atmos. Sci., 32, 25-59, https:// doi.org/10.1175/1520-0469(1975)032<0025:BAOATC>2.0.CO;2. 
Liu, Y., D. Zhang, and M. Yau, 1997: A multiscale numerical study of Hurricane Andrew (1992). Part I: Explicit simulation and verification. Mon. Wea. Rev., 125, 3073-3093, https://doi.org/ 10.1175/1520-0493(1997)125<3073:AMNSOH >2.0.CO;2.

Mlawer, E. J., S. J. Taubman, P. D. Brown, M. J. Iacono, and S. A. Clough, 1997: Radiative transfer for inhomogeneous atmosphere: RRTM, a validated correlated-k model for the longwave. J. Geophys. Res., 102, 16663-16682, https://doi.org/ 10.1029/97JD00237.

Nolan, D. S., Y. Moon, and D. P. Stern, 2007: Tropical cyclone intensification from asymmetric convection: Energetics and efficiency. J. Atmos. Sci., 64, 3377-3405, https://doi.org/ 10.1175/JAS3988.1.

Ohno, T., and M. Satoh, 2015: On the warm core of a tropical cyclone formed near the tropopause. J. Atmos. Sci., 72, 551-571, https://doi.org/10.1175/JAS-D-14-0078.1.

Qiu, X., and Z.-M. Tan, 2013: The roles of asymmetric inflow forcing induced by outer rainbands in tropical cyclone secondary eyewall formation. J. Atmos. Sci., 70, 953-974, https:// doi.org/10.1175/JAS-D-12-084.1.

_,- , and Q. Xiao, 2010: The roles of vortex Rossby waves in hurricane secondary eyewall formation. Mon. Wea. Rev., 138, 2092-2019, https://doi.org/10.1175/2010MWR3161.1.

Rozoff, C. M., W. H. Schubert, and J. P. Kossin, 2008: Some dynamical aspects of tropical cyclone concentric eyewalls. Quart. J. Roy. Meteor. Soc., 134, 583-593, https://doi.org/10.1002/qj.237.

, D. S. Nolan, J. P. Kossin, F. Zhang, and J. Fang, 2012: The roles of an expanding wind field and inertial stability in tropical cyclone secondary eyewall formation. J. Atmos. Sci., 69 , 2621-2643, https://doi.org/10.1175/JAS-D-11-0326.1.

Schubert, W. H., and J. J. Hack, 1982: Inertial stability and tropical cyclone development. J. Atmos. Sci., 39, 1687-1697, https:// doi.org/10.1175/1520-0469(1982)039<1687:ISATCD > 2.0.CO;2.

- C. M. Rozoff, J. L. Vigh, B. D. McNoldy, and J. P. Kossin, 2007: On the distribution of subsidence in the hurricane eye. Quart. J. Roy. Meteor. Soc., 133, 595-605, https://doi.org/10.1002/qj.49.

Schwartz, M. J., J. W. Barrett, P. W. Fieguth, P. W. Rosenkranz, M. S. Spina, and D. H. Staelin, 1996: Observations of thermal and precipitation structure in a tropical cyclone by means of passive microwave imagery near $118 \mathrm{GHz}$. J. Appl. Meteor., 35, 671-678, https://doi.org/10.1175/1520-0450(1996)035<0671:OOTAPS > 2.0.CO;2.

Sitkowski, M., J. P. Kossin, and C. M. Rozoff, 2011: Intensity and structure changes during hurricane eyewall replacement cycles. Mon. Wea. Rev., 139, 3829-3847, https://doi.org/10.1175/ MWR-D-11-00034.1.

— — — - - and J. Knaff, 2012: Hurricane eyewall replacement cycle thermodynamics and the relict inner eyewall circulation. Mon. Wea. Rev., 140, 4035-4045, https://doi.org/ 10.1175/MWR-D-11-00349.1.

Smith, R. K., 1980: Tropical cyclone eye dynamics. J. Atmos. Sci., 37, 1227-1232, https://doi.org/10.1175/1520-0469(1980)037<1227: TCED $>2.0 . C O ; 2$.

Stern, D. P., and D. S. Nolan, 2012: On the height of the warm core in tropical cyclones. J. Atmos. Sci., 69, 1657-1680, https:// doi.org/10.1175/JAS-D-11-010.1.

—_ and F. Zhang, 2013: How does the eye warm? Part I: A potential temperature budget analysis of an idealized tropical cyclone. J. Atmos. Sci., 70, 73-90, https://doi.org/10.1175/JASD-11-0329.1.

— and -2016 : The warm-core structure of Hurricane Earl (2010). J. Atmos. Sci., 73, 3305-3328, https://doi.org/10.1175/ JAS-D-15-0328.1.
Sun, Y. Q., Y. Jiang, B. Tan, and F. Zhang, 2013: The governing dynamics of the secondary eyewall formation of Typhoon Sinlaku (2008). J. Atmos. Sci., 70, 3818-3837, https://doi.org/ 10.1175/JAS-D-13-044.1.

Terwey, W. D., and M. T. Montgomery, 2008: Secondary eyewall formation in two idealized, full-physics modeled hurricanes. J. Geophys. Res., 113, D12112, https://doi.org/10.1029/ 2007JD008897.

Tyner, B., P. Zhu, J. A. Zhang, S. Gopalakrishnan, F. Marks Jr., and V. Tallapragada, 2018: A top-down pathway to secondary eyewall formation in simulated tropical cyclones. J. Geophys. Res. Atmos., 123, 174-197, https://doi.org/10.1002/2017JD027410.

Vigh, J. L., and W. H. Schubert, 2009: Rapid development of the tropical cyclone warm core. J. Atmos. Sci., 66, 3335-3350, https://doi.org/10.1175/2009JAS3092.1.

Wang, H., and Y. Wang, 2014: A numerical study of Typhoon Megi (2010). Part I: Rapid intensification. Mon. Wea. Rev., 142, 29-48, https://doi.org/10.1175/MWR-D-13-00070.1.

, C.-C. Wu, and Y. Wang, 2016: Secondary eyewall formation in an idealized tropical cyclone simulation: Balanced and unbalanced dynamics. J. Atmos. Sci., 73, 3911-3930, https:// doi.org/10.1175/JAS-D-15-0146.1.

_ - Y. Wang, J. Xu, and Y. H. Duan, 2019: The axisymmetric and asymmetric aspects of the secondary eyewall formation in a numerically simulated tropical cyclone under idealized conditions on an $f$ plane. J. Atmos. Sci., 76, 357-378, https:// doi.org/10.1175/JAS-D-18-0130.1.

Wang, X., and H. Jiang, 2019: A 13-year climatology of tropical cyclone warm-core structures from AIRS data. Mon. Wea. Rev., 147, 773-790, https://doi.org/10.1175/MWR-D-18-0276.1.

Wang, Y., 2007: A multiply nested, movable mesh, fully compressible, nonhydrostatic tropical cyclone model-TCM4: Model description and development of asymmetries without explicit asymmetric forcing. Meteor. Atmos. Phys., 97, 93-116, https://doi.org/10.1007/s00703-006-0246-z.

- 2008: Structure and formation of an annular hurricane simulated in a fully compressible, nonhydrostatic model-TCM4. J. Atmos. Sci., 65, 1505-1527, https://doi.org/10.1175/2007JAS2528.1.

- 2009: How do outer spiral rainbands affect tropical cyclone structure and intensity? J. Atmos. Sci., 66, 1250-1273, https:// doi.org/10.1175/2008JAS2737.1.

, 2012: Recent research progress on tropical cyclone structure and intensity. Trop. Cyclone Res. Rev., 1, 254-275.

Willoughby, H. E., 1998: Tropical cyclone eye thermodynamics. Mon. Wea. Rev., 126, 3053-3067, https://doi.org/10.1175/15200493(1998)126<3053:TCET>2.0.CO;2.

, J. A. Clos, and M. G. Shoreibah, 1982: Concentric eye walls, secondary wind maxima, and the evolution of the hurricane vortex. J. Atmos. Sci., 39, 395-411, https://doi.org/10.1175/ 1520-0469(1982)039<0395:CEWSWM > 2.0.CO;2.

Zhang, D.-L., and H. Chen, 2012: Importance of the upper-level warm core in the rapid intensification of a tropical cyclone. Geophys. Res. Lett., 39, L02806, https://doi.org/10.1029/ 2011 GL050578.

Zhang, F., D. Tao, Y. Q. Sun, and J. D. Kepert, 2017: Dynamics and predictability of secondary eyewall formation in sheared tropical cyclones. J. Adv. Model. Earth Syst., 9, 89-112, https:// doi.org/10.1002/2016MS000729.

Zhu, Z., and P. Zhu, 2014: The role of outer rainband convection in governing the eyewall replacement cycle in numerical simulations of tropical cyclones. J. Geophys. Res. Atmos., 119, 8049-8072, https://doi.org/10.1002/2014JD021899. 